\title{
Enhanced insulin secretion and cholesterol metabolism in congenic strains of the spontaneously diabetic (Type 2) Goto Kakizaki rat are controlled by independent genetic loci in rat chromosome 8
}

\author{
R. H. Wallis ${ }^{1}$ K. J. Wallace ${ }^{1}$ S. C. Collins ${ }^{1}$ M. McAteer ${ }^{1}$ M. Krgoud $^{1}$ - M. T. Bihoreau ${ }^{1}$ P. J. Kaisaki ${ }^{1}$ \\ D. Gauguier ${ }^{1}$
}

1 The Wellcome Trust Centre for Human Genetics, University of Oxford, Headington, Oxford, UK

\begin{abstract}
Aims/hypothesis. Genetic investigations in the spontaneously diabetic (Type 2) Goto Kakizaki (GK) rat have identified quantitative trait loci (QTL) for diabetes-related phenotypes. The aims of this study were to refine the chromosomal mapping of a QTL (Nidd/gk5) identified in chromosome 8 of the GK rat and to define a pathophysiological profile of GK gene variants underlying the QTL effects in congenics.

Methods. Genetic linkage analysis was carried out with chromosome 8 markers genotyped in a GKxBN F2 intercross previously used to map diabetes QTL. Two congenic strains were designed to contain GK haplotypes in the region of Nidd/gk5 transferred onto a Brown Norway (BN) genetic background, and a broad spectrum of diabetes phenotypes were characterised in the animals.

Results. Results from QTL mapping suggest that variations in glucose-stimulated insulin secretion in vivo, and in body weight are controlled by different chromosome 8 loci (LOD3.53; $p=0.0004$ and LOD4.19; $p=0.00007$, respectively). Extensive physiological screening in male and female congenics at 12 and 24 weeks revealed the
\end{abstract}

existence of GK variants at the locus Nidd/gk5, independently responsible for significantly enhanced insulin secretion and increased levels of plasma triglycerides, phospholipids and HDL, LDL and total cholesterol. Sequence polymorphisms detected between the $\mathrm{BN}$ and GK strains in genes encoding ApoAI, AIV, CIII and Lipc do not account for these effects.

Conclusions/interpretation. We refined the localisation of the QTL Nidd/gk5 and its pathophysiological characteristics in congenic strains derived for the locus. These congenic strains provide novel models for testing the contribution of a subset of GK alleles on diabetes phenotypes and for identifying diabetes susceptibility genes.

Electronic Supplementary Material

Supplementary material is available in the online version of this article at http://dx.doi.org/10.1007/s00125-004-1416-5

Keywords Cholesterol · Congenic strains · Genetics · Goto Kakizaki rat · Quantitative trait locus · Type 2 diabetes mellitus
Received: 14 January 2004 / Accepted: 3 March 2004

Published online: 26 May 2004

(C) Springer-Verlag 2004

D. Gauguier (®)

The Wellcome Trust Centre for Human Genetics,

University of Oxford, Roosevelt Drive, Headington,

Oxford, OX3 7BN, UK

E-mail: gdomi@well.ox.ac.uk

Tel.: +44-1865-287648

Fax: +44-1865-287533
Abbreviations: AIS, acute insulin secretion - APO, apolipoprotein · Asn, asparagin · BN, Brown Norway · cM, centimorgan $\cdot$ EST, expressed sequence tags $\cdot$ GK, Goto Kakizaki · GLM, general linear model · LIPC, hepatic lipase $\cdot$ LOD, logarithm of odds ratio $\cdot \mathrm{Mb}$, megabase . OLETF, Otsuka Long-Evans Tokushima fatty · PL, phospholipids - QTL, quantitative trait locus/loci · RFPW, retroperitoneal fat pad weight $\cdot$ SHR, spontaneously hypertensive rat - SNP, single nucleotide polymorphism . SPG, sum of plasma glucose $\cdot$ SPI, sum of plasma insulin $\cdot$ TC, total cholesterol $\cdot \mathrm{TG}$, triglycerides $\cdot \mathrm{UTR}$, untranslated region 


\section{Introduction}

Multiple genetic and environmental factors contribute to the complex pathophysiological heterogeneity of the most common forms of human Type 2 diabetes mellitus. Although genetic linkage studies in humans have identified numerous chromosomal regions cosegregating with diabetes phenotypes [1], convincing evidence for the identification of underlying causal gene variants is sparse, and may be limited to the gene encoding Calpain 10 [2]. Investigations in rodent models of spontaneous diabetes represent an alternative for characterising essential components involved in glucose homeostasis and for identifying diabetes candidate genes and gene pathways.

Among the best-characterised animal models of Type 2 diabetes, the inbred Goto Kakizaki (GK) rat strain displays both impaired insulin secretion and insulin resistance from an early age but does not become obese $[3,4]$. Genetic studies in the GK rat have identified broad chromosomal regions (quantitative trait loci; QTL) that contain gene variants controlling glucose homeostasis and insulin secretion [5, 6]. Among these, the QTL Nidd/gk5 was detected in a poorly characterised region of rat chromosome 8 in an intercross between the GK rat and rats of the non-diabetic Brown Norway (BN) strain [6]. The QTL was primarily associated with glucose-induced insulin secretion and, to a lesser extent, with body weight. Poor mapping resolution and modest phenotypic effects are the main drawbacks of QTL detected in classical studies based on linkage analysis between genotypes and quantitative variations of a limited number of traits in a two-generation cross [7].

Congenic strains are powerful new tools that are now widely used to progress from broad statistical estimates of a genetic effect in hybrids of a segregating population, to an accurate characterisation of a chromosomal region carrying disease susceptibility genes $[8,9,10]$. A congenic strain is a novel inbred model which is identical to the parental strain except that a selected chromosomal segment has been replaced by the corresponding region from the disease-resistant or disease-susceptible strain. Panels of congenic strains represent essential novel experimental models for (i) providing biological evidence for the existence of a QTL, (ii) fine mapping the underlying disease gene(s) and (iii) profiling their pathophysiological consequences prior to gene cloning and mutation detection.

In order to refine the mapping of the locus Nidd/gk5 originally described in the GKxBN F2 cross [6], we initially carried out linkage mapping analysis using an improved version of the genetic map of rat chromosome 8 constructed in the cross [11]. We subsequently derived congenic strains designed to investigate the phenotypic effects of GK alleles in regions of the QTL when transferred onto the genetic background of the $\mathrm{BN}$ strain. Extensive phenotypic analyses in congenics confirmed the existence of GK alleles at the QTL, al- tering insulin secretion and body weight, as detected in the original GKxBN cross. Furthermore, we were able to demonstrate that gene variants in this chromosomal region alter plasma cholesterol metabolism.

\section{Materials and methods}

\section{Animals}

The GKxBN F2 cross ( $n=138)$, originally derived for detecting QTL underlying diabetes variables, was used to fine map the locus Nidd/gk5 previously identified in rat chromosome 8 in this population [6]. Breeding and phenotype screening, including mainly glucose tolerance, in vivo glucose-induced insulin secretion, body weight and adiposity, have been previously described in detail [6].

For the production of congenic strains, a colony of GK rats was initiated in Oxford in 1995 using breeders obtained from the colony derived in Paris (CNRS URA 307, Paris, France). $\mathrm{BN}$ rats were obtained from a commercial supplier (Charles River Laboratories, Margate, UK). All rats had free access to water and standard laboratory chow pellets (ERB, Whitam, UK), and were maintained on a 12-h light and dark cycle. In all breeding experiments, pregnancy was detected by abdominal palpation at day 13 of gestation onwards. Pups were weaned at 21 days. All procedures were carried out in accordance with our institutional guidelines.

Microsatellite marker-assisted production of the congenic strains

The construction of congenic strains was specifically designed to introgress GK alleles in two overlapping regions of rat chromosome 8, covering part of the original QTL Nidd/gk5, onto the genetic background of the BN strain. Initially, a population of GKxBN F1 animals was derived from a male GK rat bred to several female $\mathrm{BN}$ rats. The first backcross breeding to the $\mathrm{BN}$ strain was performed by caging a male $\mathrm{F} 1$ rat with several $\mathrm{BN}$ females. The $\mathrm{BN}$ allele of the $\mathrm{X}$ chromosome was therefore fixed in two successive generations, and microsatellite screening (see below) of this chromosome was not required. Subsequent backcross breedings to $\mathrm{BN}$ rats were carried out using either male or female backcross hybrids. Final inbreeding was performed by brother-sister mating in order to fix the homozygous GK alleles at the selected loci.

At each backcross generation, the genome of the hybrids was screened using a collection of microsatellite markers that span the 20 autosomes of the rat genome and that are known to exhibit allele variations between GK and BN strains [11]. The panel of markers was optimised in each successive generation in order to verify the integrity of the GK homozygous haplotype at the locus Nidd/gk5, and to monitor the elimination of GK alleles from the genetic background, particularly in regions containing other QTL previously identified in the GKxBN and GKxF344 intercrosses [5, 6].

Records of successive breedings and subsequent genetic and physiological screening of the congenics were stored in our database specifically designed for the production and characterisation of congenic strains [12].

\section{Genotype determination of backcross progenies}

Genomic DNA of rat progeny was prepared from tail tips. The PCR reactions were carried out with $50 \mathrm{ng}$ of genomic DNA as 
previously described [11]. Primer sequences, PCR conditions and mapping information for all rat microsatellite markers are available from our public database (http://www.well.ox.ac.uk/ rat_mapping_resources). Oligonucleotides were synthesised commercially by Sigma-Genosys Biotechnologies (Pampisford, UK).

\section{Phenotypic characterisation of congenic strains}

All physiological tests were performed in both male and female rats and repeated at 12 and 24 weeks of age. Glucose tolerance and insulin secretion tests were performed using the protocol applied to the phenotypic screening of the GKxBN F2 hybrids [6]. Briefly, rats in the post-absorptive state were anaesthetised by injection of $95 \mathrm{mg} / \mathrm{kg}$ body weight i.p. ketamine hydrochloride (Ketalar, Parke-Davies, UK) and injected via the saphenous vein with $0.8 \mathrm{~g}$ glucose $/ \mathrm{kg}$ body weight. Blood samples were collected into heparinised tubes before glucose injection and after 5, 10, 15, 20 and 30 min. Samples were centrifuged and the plasma was separated and stored at $-80{ }^{\circ} \mathrm{C}$ for subsequent measurement of glucose and immunoreactive insulin concentrations.

Following a 16 to 18 -h fast, blood samples were collected, and plasma was obtained by centrifugation and stored at $-80{ }^{\circ} \mathrm{C}$ until assays were performed for the quantification of lipid and lipoprotein concentrations.

Anchoring congenic intervals in the rat genome sequence and comparative genome analysis

Known microsatellite markers, including markers designed in known genes, already localised in the genetic map of rat chromosome 8 in the GKxBN F2 cross, were searched for in the rat genome sequence. Rat genes assigned to the targeted chromosomal regions in congenics were subsequently used to search for orthologues in human and mouse genome databases (http://ensembl.ebi.ac.uk) in order to define homology relationships between the rat, mouse and human genomes in the region of Nidd/gk5. We used both existing comparative gene maps between human, mouse and rat genomes [11, 13], and annotation of the rat genome sequence (http://ensembl.ebi.ac.uk) to define anchor points between the genetic map of rat chromosome 8 and human, rat and mouse genome sequences.

\section{Candidate genes sequence screening}

PCR and sequencing. Genomic DNA from GK and BN rats was screened for sequence variants in hepatic lipase (Lipc), ApoAIV and ApoCIII. Primers were designed to screen the exons from the genomic sequences that were available, with the following accession numbers: J02588 for ApoAIV, and J02596 for ApoCIII. For Lipc, bacterial artificial chromosome sequences were downloaded from the Baylor College of Medicine rat genome project website (http://www.hgsc.bcm.tmc.edu/ projects/rat/). Excess primers were removed from the PCR products, and then the amplicons were sequenced on an Applied Biosystems 377 DNA sequencer using the BigDye version 1 dye terminator kit (Applied Biosystems, Warrington, $\mathrm{UK}$ ). Information on primer sequences and PCR products analysed for sequence variants are publicly available from our rat data repository (http://www.well.ox.ac.uk/rat_mapping_ resources) and can be viewed online (via ESM [electronic supplementary material] link in online version of this article).
Analytical methods

Plasma concentrations of glucose, total cholesterol (TC), HDL$\mathrm{C}$, LDL-C, triglycerides and phospholipids were determined using diagnostic enzymatic/colorimetric kits (ABX, Shefford, UK) on a Cobas Mira Plus automatic analyser (ABX, Shefford, UK). Values for VLDL-C were obtained by subtracting the sum of HDL-C and LDL-C from TC, since most non-HDL-C is in the VLDL fraction in rodents [14]. Plasma levels of NEFA were also quantified on the Cobas Mira Plus using an enzymatic/colorimetric kit from Wako (Alpha Laboratories, Eastleigh, UK). Plasma insulin concentrations were measured using ELISA for rat insulin (Mercodia, Uppsala, Sweden).

\section{Statistical analyses}

Fine QTL mapping in the GKxBN F2 cross. A subset of the microsatellite markers used to construct a dense consensus genetic map of rat chromosome 8 in the GKxBN F2 cross [11] were selected to carry out genetic linkage analyses with diabetes variables. Linkage between genotypes and diabetes-related phenotypes standardised for both sex and cross effects as previously described [6] was initially evaluated by an ANOVA test followed by a permutation test $(n=10,000)$ [15]. Interval mapping was subsequently performed using MapMaker/QTL computer package [16].

Analysis of phenotypes quantified in congenic strains. Data from all phenotypes collected in the animals were analysed using the statistical package, SPSS version 11.5. The Univariate General Linear Model (GLM), which allows the selection of covariates to account for variance that is not due to the dependent variable, was used to analyse phenotypes quantified in congenic and $\mathrm{BN}$ rats. A Bonferroni post hoc test was used to assess differences between the control strain and the different congenic lines, as well as between congenic strains. A $p$ value of less than 0.05 was considered to be statistically significant for pairwise comparisons.

\section{Results}

Fine QTL mapping for diabetes-related traits in the GKxBN F2 cohort

Genotype data derived from a subset of 20 microsatellite markers selected among the 92 chromosome 8 markers recently mapped in the GKxBN F2 cohort [11] were used for refining the location of Nidd/gk5. These markers identified individual genetic positions with an average spacing of 4.5 centimorgan (cM) between adjacent loci. High-resolution genetic mapping of this chromosome in the GKxBN F2 region allowed discrimination of two QTL effects on glucose-induced insulin secretion and body weight (Fig. 1). Evidence of significant linkage was found between marker locus D8Got99 and acute insulin secretion in response to glucose (5 and $10 \mathrm{~min}$ after i.v. glucose injection) during the IVGTT (maximum LOD3.53, $p=0.0004$ ). The strongest evidence of linkage to body weight in F2 hybrids was detected at marker locus D8Got144 (LOD4.19, $p=0.00007$ and LOD3.93, $p=0.0001$ at 12 and 24 weeks, respectively) which maps $16 \mathrm{cM}$ away from D8Got99. These QTL account for up to $11.2 \%$ 


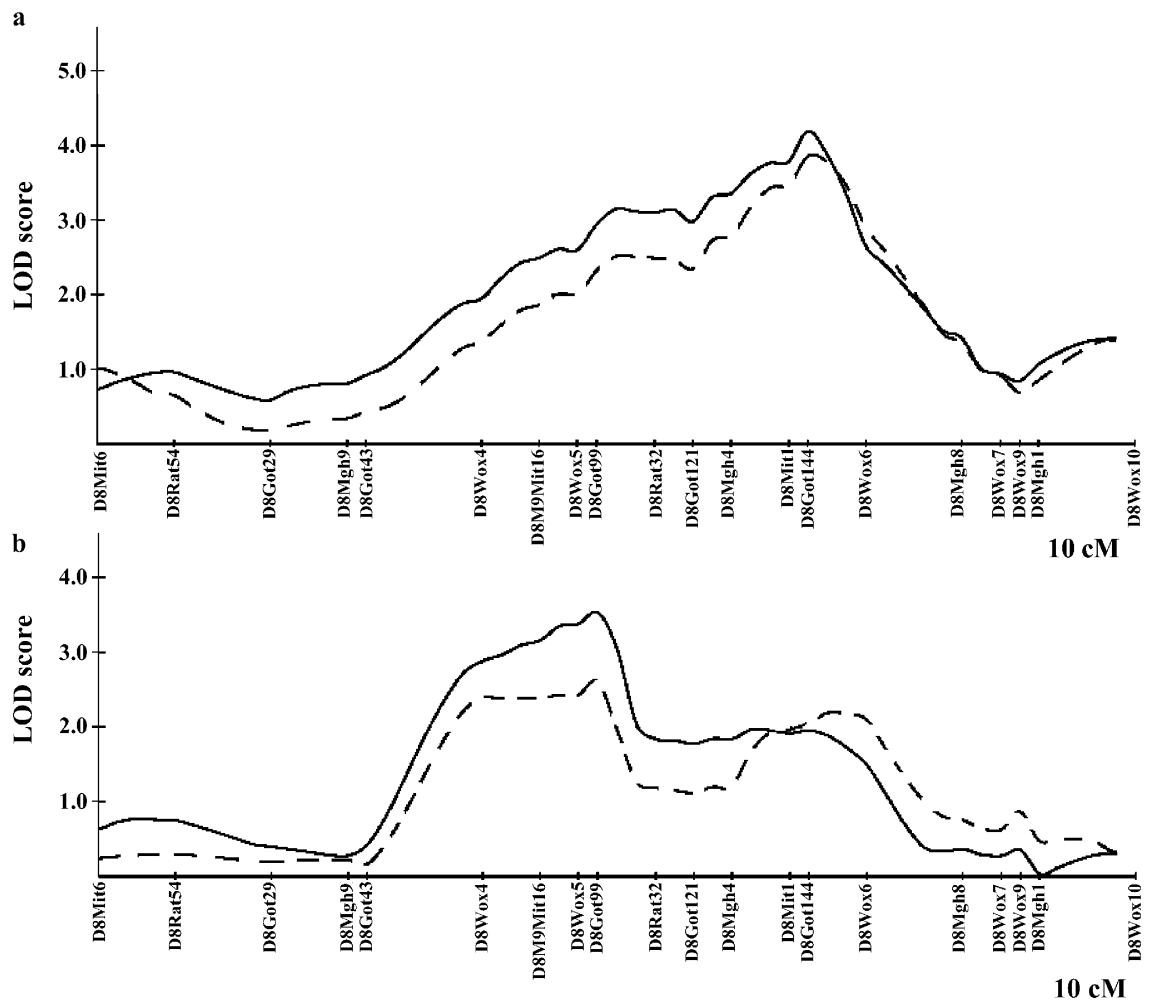

Fig. 1. QTL maps for (a) body weight at 12 (unbroken line) and 24 (broken line) weeks, and (b) insulin secretion in the GKxBN F2 cross. LOD plots are shown for acute insulin secretion $5 \mathrm{~min}$ after glucose injection (unbroken line) and for the sum of plasma insulin (broken line). The refined QTL map of the locus Nidd/gk5 in the GKxBN F2 cross was constructed using a simplified version of the genetic map generated in the cross [11] (http://www.well.ox.ac.uk/rat_mapping_resources). Genetic lengths are in cM (Kosambi)

and $13 \%$ of the variance of insulin secretion and body weight respectively in the cross.

No evidence of significant linkage was found between chromosome 8 loci and phenotypes related to acute glucose response to glucose challenge, cumulative glycaemia during the IVGTT or adiposity.

Rats of the F2 cohort carrying the GK homozygous genotype at marker locus D8Got99 exhibited significantly enhanced in vivo glucose-induced insulin secretion (+28\%) when compared with rats carrying the BN homozygous genotype (Table 1). Although fasting insulin was more elevated in rats homozygous at the locus for GK alleles than in those homozygous for BN alleles, the effect was not statistically significant.

Genetic characterisation of congenic strains derived for $\mathrm{Nidd} / \mathrm{gk} 5$

We derived two congenic strains, BN.GK8a (BN. GK-D8Rat29/D8Got130) and BN.GK8b (BN.GKD8Got302/D8Got130), which contain GK haplotypes in $6.9 \mathrm{cM}$ and $24.3 \mathrm{cM}$ chromosomal regions of chro-

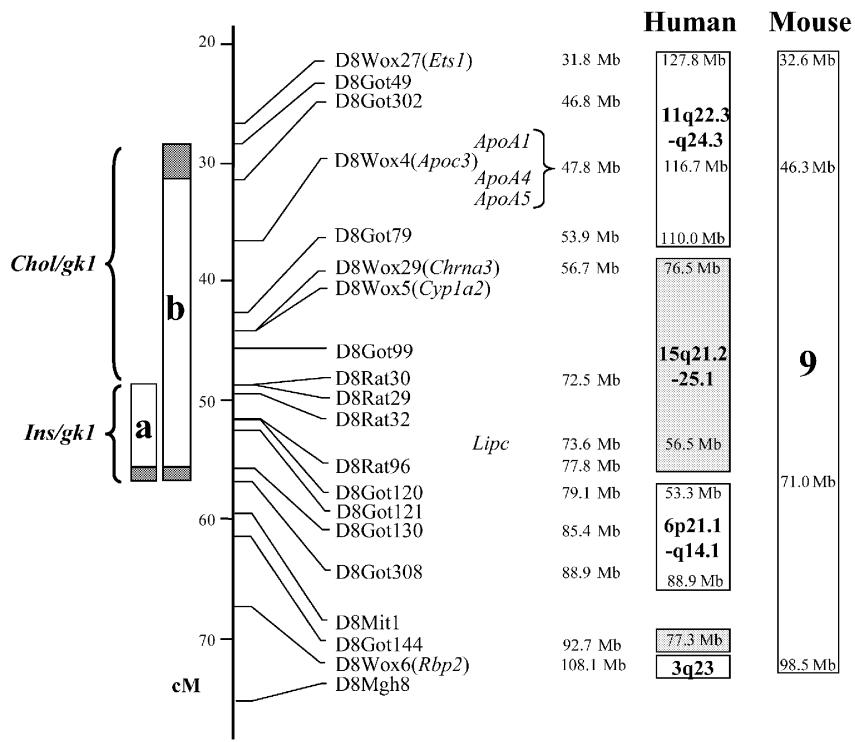

Fig. 2. Targeted chromosomal intervals in congenic strains and comparative gene maps at the original locus Nidd/gk5. Open bars represent the chromosomal region carrying the GK haplotype in congenics. Solid bars at the end of the congenic segments are regions of crossover where genotype is unknown. Comprehensive genetic maps in the GKxBN F2 cross and full results from comparative genome mapping in the regions of Ins/gkl and Chol/gkl are available on our public database (http://www.well.ox.ac.uk/rat_mapping_resources)

mosome 8 respectively, introgressed onto the genetic background of the BN strain (Table 2, Fig. 2). The chromosomal regions of the QTL Nidd/gk5 containing GK alleles in the congenic strains, BN.GK8a and BN.GK8b, were screened with 6 and 13 markers re- 
Table 1. Correlations between genotypes at marker loci D8Got99 and D8Got144 and variables of insulin secretion and body weight in GKxBN F2 rats

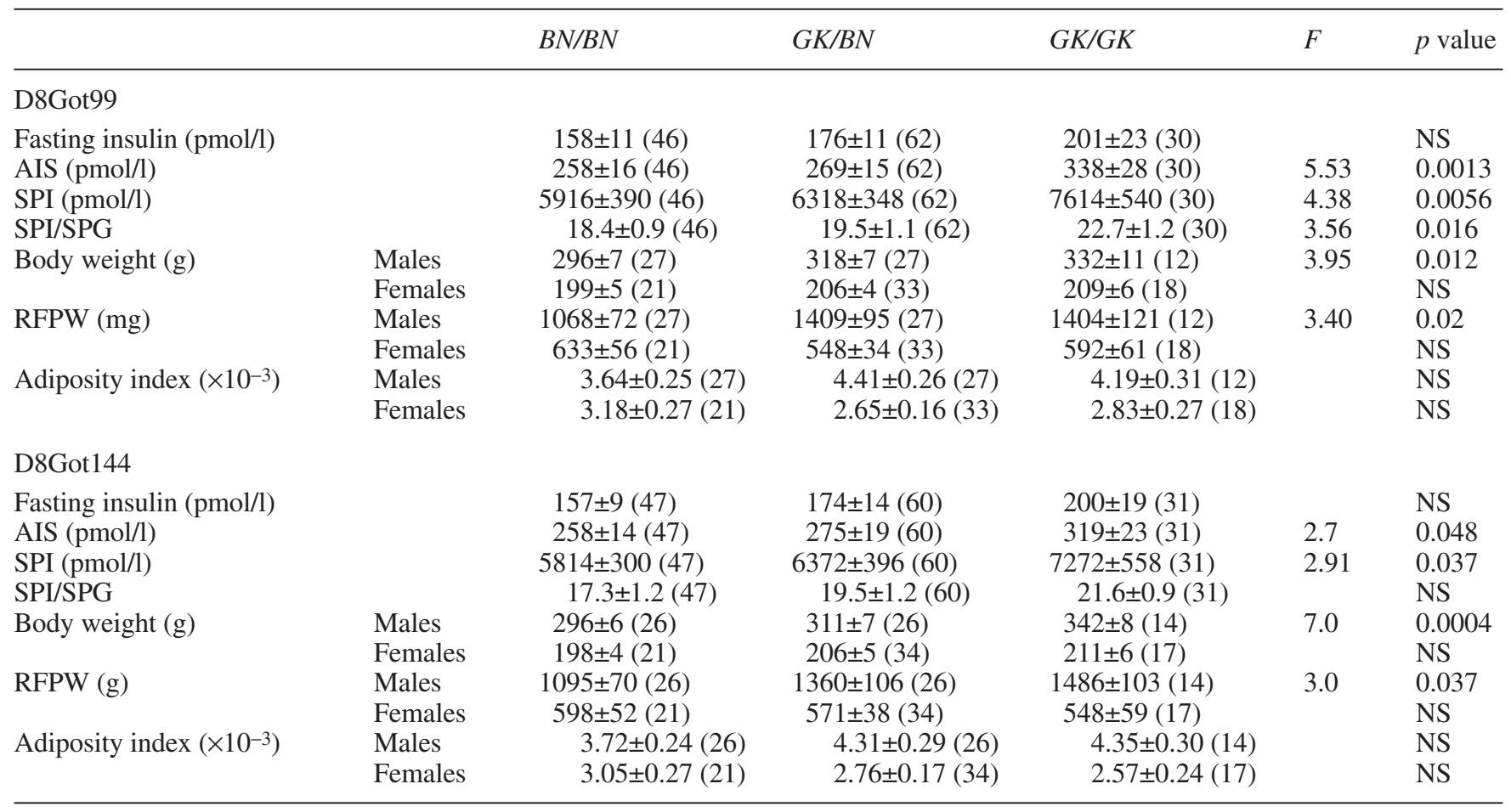

Plasma insulin concentration 5 min after intravenous glucose stimulation was used as an index of acute insulin secretion (AIS). Sums of plasma insulin (SPI) and glucose (SPG) during the test were used to evaluate the overall insulin secretory response to glucose. The ratio of retroperitoneal fat pad weight (RFPW) : body weight was used as an index of adiposity. For

all phenotypes, means \pm SE were calculated for each genotype at the locus. $B N / B N$, homozygous for the $\mathrm{BN}$ allele; $G K / B N$, heterozygous; $G K / G K$, homozygous for the GK allele. Number of observations is given in parentheses. Following regression of insulin secretion phenotypes for both sex and cross, analysis of variance was applied to test for linkage

Table 2. Congenic lines derived for the QTL Nidd/gk5 and genetic size of regions containing GK homozygous genotypes

\begin{tabular}{|c|c|c|c|c|c|}
\hline $\begin{array}{l}\text { Congenic } \\
\text { line }\end{array}$ & Maximum interval (cM) ${ }^{\mathrm{a}}$ & Minimum interval $(\mathrm{cM})^{\mathrm{a}}$ & $\begin{array}{l}\text { Markers } \\
\text { typed }^{\mathrm{b}}\end{array}$ & $\begin{array}{l}\text { Resolution } \\
(\mathrm{cM})^{\mathrm{c}}\end{array}$ & $\begin{array}{l}\text { Generation } \\
\text { at inbreedingd }\end{array}$ \\
\hline BN.GK8a & D8Rat30-D8Got308 (8.1) & D8Rat29-D8Got130 (6.9) & $247(127)$ & $6.23(6.47)$ & 8 \\
\hline
\end{tabular}

a Chromosome 8 marker loci that were homozygous for the $\mathrm{BN}$ or the GK allele were used to define the maximum and minimum congenic interval respectively, containing GK haplotypes. The length of the congenic region in parentheses was obtained in the linkage map of the GKxBN F2 cross [11]. ${ }^{\mathrm{b}}$ Total number of markers used for the genetic screening of the QTL Nidd/gk5 and the genetic background in progenies of backcross breedings. The number of markers used to

spectively, which were used to determine the minimal genetic length of the congenic intervals. The dense marker coverage of these regions (average spacing between markers of $1.1 \mathrm{cM}$ in BN.GK8a and $1.8 \mathrm{cM}$ in BN.GK8b congenics) was used to verify the integrity of the GK haplotype in the congenic intervals.

A total of 247 (BN.GK8a) and 234 (BN.GK8b) microsatellite markers were used to screen the 20 rat autosomes in the 8 (BN.GK8a) and 6 (BN.GK8b) succes- screen chromosomal segments containing GK QTL, including Nidd/gk5, is shown in parentheses. ${ }^{\mathrm{c}}$ Average spacing between markers used for the genetic characterisation of the congenics. Results from the screening of chromosomes containing known GK QTL are shown in parentheses. ${ }^{d}$ Backcross generation at which GK alleles were eliminated from the genetic background and inbreeding was initiated

sive $(\mathrm{GKxBN}) \mathrm{xBN}$ backcross generations that were required for the elimination of GK alleles throughout the genetic background of the congenic strains prior to brother-sister matings (inbreeding; Table 2). Approximately half of these markers were chosen to screen known diabetes QTL identified in GKxBN and GKxF344 intercrosses, and to monitor the exclusion of GK alleles at these loci in the congenics [5, 6]. Overall, the genome of rat progeny was scanned with an average spacing of $<7 \mathrm{cM}$ between loci. 


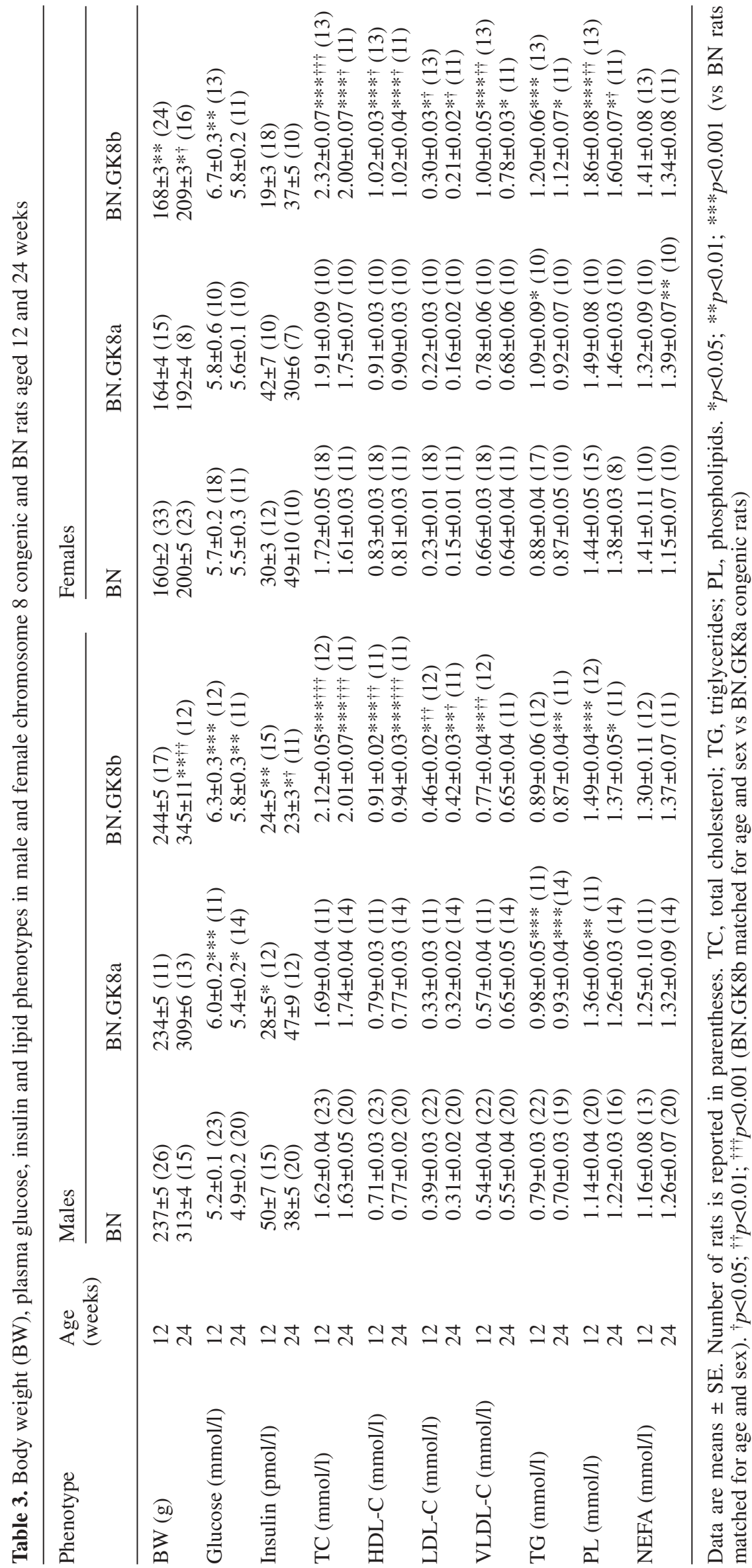



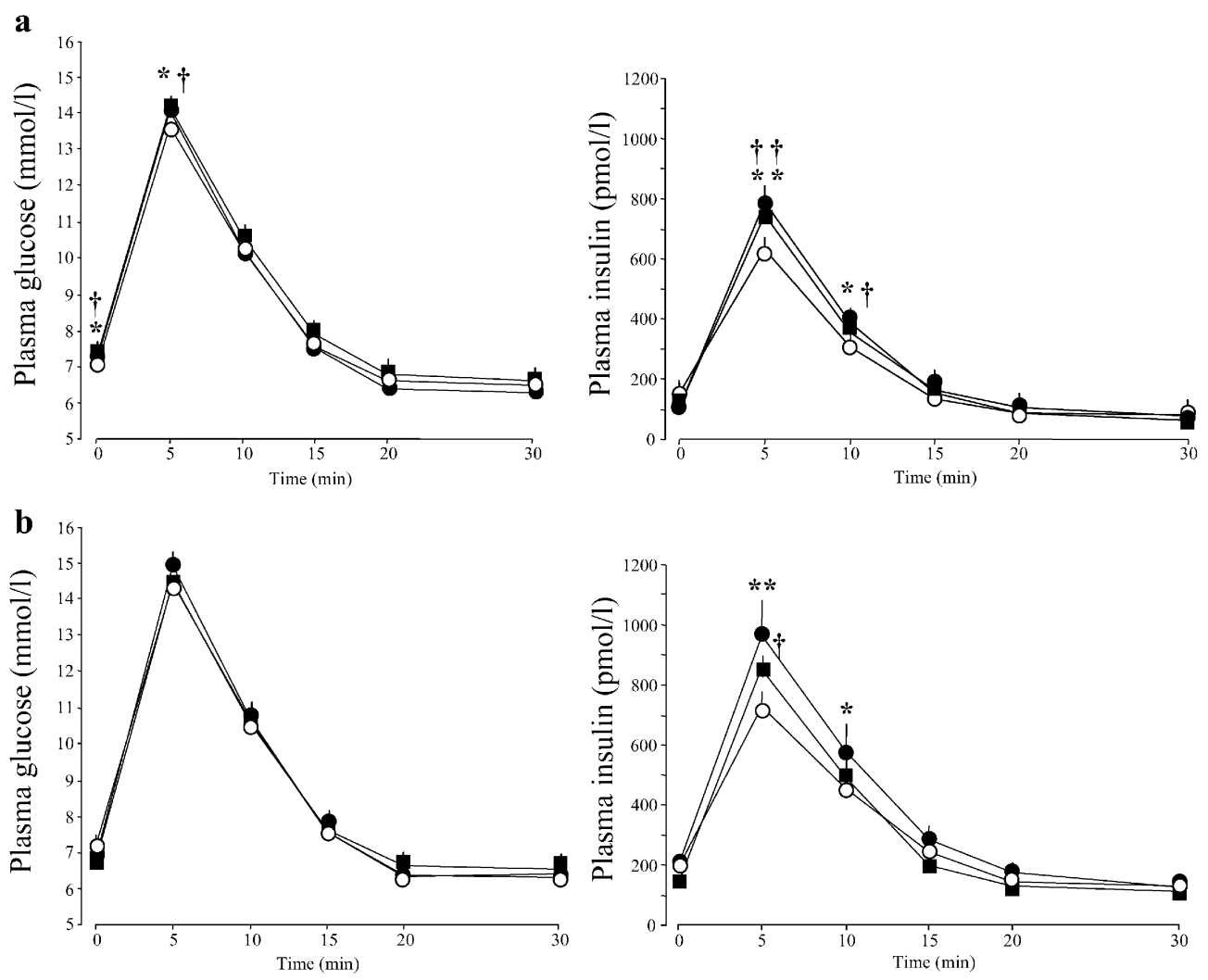

Fig. 3. Effects of intravenous glucose injection on plasma glucose and immunoreactive insulin concentrations at 12 (a) and 24 (b) weeks in BN rats (white circles) and rats of congenic strains BN.GK8a (black circles) and 8b (black squares). Results are shown as means $\pm \mathrm{SE} ; * p<0.05, * * p<0.01$ (BN.GK8a congenic rats vs $\mathrm{BN}$ controls); $\uparrow p<0.05, \dagger \dagger p<0.01$ (BN.GK8b congenic rats vs $\mathrm{BN}$ controls)

Phenotypic characterisation of congenic strains BN.GK8a and BN.GK8b

Body weight and fasting glycaemia and insulinaemia. At 12 and 24 weeks, body weight was marginally higher in female BN.GK8b rats than in BN controls (Table 3). In contrast, 24-week-old male BN.GK8b rats were significantly heavier than BN.GK8a rats matched for age and sex $(p=0.006)$ and than $\mathrm{BN}$ controls $(p=0.007)$. No differences in body weight were observed between BN.GK8a and $\mathrm{BN}$ rats at either 12 or 24 weeks.

Fasting plasma glucose levels were significantly higher in 12 and 24-week-old male BN.GK8a rats than in $\mathrm{BN}$ rats, while no differences were observed between female BN.GK8a and BN rats (Table 3 ). Fasting glycaemia was significantly increased in 12 and 24-week-old BN.GK8b males and in 12-week-old females only, when compared with BN controls matched for age and sex.

In BN.GK8a congenic rats, fasting insulinaemia was significantly lower than in $\mathrm{BN}$ rats only, in males at 12 weeks. Fasting insulinaemia was lower in both

12 and 24-week-old BN.GK8b rats than in BN rats, but this effect was statistically significant in males only.

Glucose tolerance. Basal plasma glucose levels were higher in 12-week-old BN.GK8a and BN.GK8b rats than in $\mathrm{BN}$ rats $(p=0.01$ and $p=0.02$ respectively; Fig. 3a). Although hyperglycaemia was observed when data from males and females at 12 weeks were combined, the effect was significant $(p<0.05)$ only in males (data not shown). Basal glycaemia was similar in the three groups of rats at 24 weeks (Fig. 3b). In 12week-old animals, glycaemia 5 min after glucose injection was significantly higher in BN.GK8a and BN.GK8b rats than in $\mathrm{BN}$ controls ( $p=0.02$; Fig. $3 \mathrm{a}$ ). Glycaemia in BN.GK8b rats tended to be higher than in BN.GK8a and BN rats at further time points, but differences were not statistically significant. At 24 weeks, the three groups of rats had similar levels of glycaemia during the IVGTT.

Glucose-induced insulin secretion. Plasma insulin levels before glucose injection were similar in congenic and control rats at both 12 and 24 weeks (Fig. 3). Insulin secretion in response to glucose was markedly enhanced in both 12 and 24-week-old BN.GK8a and BN.GK8b congenic rats when compared with agematched BN controls. The most significant effects were observed $5 \mathrm{~min}$ after the glucose challenge in 12 -week-old BN.GK8a and BN.GK8b rats $(p<0.01$ when compared with $\mathrm{BN}$ rats) and resulted in $>20 \%$ increase in the peak insulin response to glucose in 
Table 4. Sequence variations between BN and GK strains in genes encoding ApoAIV, ApoCIII and Lipc

\begin{tabular}{lllllll}
\hline Gene & GenBank & Position & Nucleotide & BN & GK & Amino acid \\
\hline Lipc & NM_012597 & Exon 5 & 624 & C & T & Asn/Asn \\
ApoAIV & J02588 & 5'UTR & 212 & A & G & - \\
& & 5'UTR & 248 & G & A & - \\
& & Intron 2 & 1301 & C & T & - \\
& & Intron 2 & 1368 & T & C & Asn/Asn \\
ApoCIII & Exon 3 & 1846 & C & T & - \\
& & J'UTR & 2600 & G & C & - \\
\hline
\end{tabular}

Asn, asparagin

congenics when compared with controls (Fig. 3a). At 24 weeks, the enhancement of acute insulin response to glucose $5 \mathrm{~min}$ after the glucose challenge was highly significant in BN.GK8a rats $(p<0.01)$ and marginally significant in BN.GK8b rats $(p=0.04)$ when compared with age-matched $\mathrm{BN}$ controls.

Plasma lipid concentrations. No major differences between BN.GK8a and $\mathrm{BN}$ rats were observed for the plasma concentrations of TC, HDL-C, LDL-C, VLDL-C and NEFA (Table 3). The level of plasma triglycerides (TG) was significantly higher in BNGK8a than in $\mathrm{BN}$, in males aged 12 and 24 weeks ( $p=0.0008$ and $p=3 \times 10^{-5}$ respectively) and in females aged 12 weeks $(p=0.031)$, but the effect was not observed in female BN.GK8a aged 24 weeks. The concentrations of plasma phospholipids (PL) and NEFA were also higher in BN.GK8a female rats at 12 and 24 weeks respectively than in $\mathrm{BN}$ controls matched for age and sex.

Plasma levels of TC were significantly greater in 12 and 24-week-old BN.GK8b rats than age-matched $\mathrm{BN}$ rats both in males $\left(p=9 \times 10^{-8}\right.$ and $p=7 \times 10^{-5}$ respectively) and females ( $p=3 \times 10^{-6}$ and $p=1 \times 10^{-3}$ respectively; Table 3). At both 12 and 24 weeks, the elevated levels of plasma TC in the BN.GK8b congenic rats were associated with a strongly significant enhancement of HDL-C, LDL-C and PL concentrations when compared with age-matched $\mathrm{BN}$ rats. This effect was strongly significant in both males and females. The concentrations of plasma VLDL-C were increased in male and female BN.GK8b rats when compared with the BN, but this effect was not significant in males at 24 weeks. Significantly higher plasma TG values were observed in BN.GK8b rats than in $\mathrm{BN}$ in males at 24 weeks $(p=0.0013)$ and in both 12 and 24week-old females ( $p=0.0002$ and $p=0.0113$ respectively). No differences were observed in NEFA concentrations between $\mathrm{BN}$.GK8b and $\mathrm{BN}$ rats.

Plasma concentrations of TC, HDL-C and LDL-C were also significantly higher in both male and female BN.GK8b congenic rats at 12 and 24 weeks than in BN.GK8a rats matched for age and sex. Both male and female BN.GK8b congenic rats at 12 weeks exhibited significantly increased concentrations of plasma VLDL-C when compared with BN.GK8a rats, but this effect was not replicated in 24-week-old rats. Plasma concentrations of PL were higher in BN.GK8b rats than in BN.GK8a rats, but this effect was statistically significant in females only.

\section{Comparative genome analysis}

We specifically carried out in silico comparative genome mapping for the chromosomal interval introgressed in BN.GK8b congenics. This region was initially integrated in the rat genomic sequence using 19 markers localised both in the draft sequence and in the congenic interval as defined in the genetic map previously constructed in the GKxBN F2 cross [11] (Fig. 2). Three markers designed in the sequence of known genes were used to define initial anchor points between the rat congenic interval and the human genome sequence. The genomic length covering the entire congenic interval $(24.3$ to $28.5 \mathrm{cM})$ in the strain BN.GK8b is 42.1 megabase (Mb). It contains 94 known genes and approximately 370 expressed sequence tags (EST) which were used to refine homology relationships with regions of mouse chromosome 9 and human chromosomes 11q22.3-q24.3 (17.8 Mb), $15 q 21.2-25.1(20 \mathrm{Mb})$ and 6p21.1-q14.1 (35.6 Mb). GK haplotypes shared by BN.GK8a and BN.GK8b strains, which are involved in the regulation of insulin secretion (QTL1), correspond to a 12.9-16.4 Mb genomic segment annotated for 31 genes and 74 ESTs in the rat. It is conserved with human chromosomes 15q21.2-22.2 and 6p21.1-q14.1. Full results from comparative genome analysis are available on our public database (http://www.well.ox.ac.uk/rat_mapping_ resources/).

Sequence variant analysis

Comparing GK and $\mathrm{BN}$ rats (Table 4), one silent single nucleotide polymorphism (SNP) was found in 
exon 5 of Lipc (624T/C; N207N). In ApoAIV, a synonymous SNP was observed in exon 3 (1846C/T; N206N), and an additional SNP was found in the 3'UTR $(2600 \mathrm{C} / \mathrm{G})$. For ApoCIII, no SNPs were found in the coding region, but one was present in the $5^{\prime}$-UTR $(404 \mathrm{C} / \mathrm{T})$.

\section{Discussion}

In the present study we have refined the genetic mapping of the QTL Nidd/gk5 linked to enhanced insulin secretion and increased body weight in a GKxBN F2 cross [6], and provided confirmatory evidence for its existence in congenic strains. Results from the characterisation of congenics, designed to carry different GK haplotypes in regions of Nidd/gk5 introgressed onto the genetic background of the BN strain, showed that different GK alleles at the QTL independently contribute to significantly enhanced glucose-induced insulin secretion and increased plasma lipid levels. These congenic strains provide powerful new models for investigating the pathophysiological consequences of individual diabetes polygenes.

The existence of the QTL Nidd/gk5 in the GKxBN F2 cross was originally evidenced by significant linkage between insulin secretion variables, and only two genetic markers known to map to rat chromosome 8 [6]. The saturation of the linkage map of this chromosome in the cross [11] allowed us to carry out interval mapping and refine the position of loci linked to body weight and insulin secretion. The peaks of genetic linkage to these variables map $16 \mathrm{cM}$ apart, suggesting an involvement of different genes in their control. Improvements in the QTL maps also allowed direct comparisons with diabetes or obesity susceptibility loci detected in other rat strains. The locus Obs3, which is linked to obesity phenotypes in a cross derived from the Otsuka Long-Evans Tokushima fatty (OLETF) rat model for Type 2 diabetes and obesity and F344 controls, coincides almost exactly with the region of Nidd/gk5 linked to body weight [17]. Other QTL for fasting plasma TG in the OLETF rat [18] and plasma HDL2 cholesterol and PL levels in a recombinant inbred panel derived from the spontaneously hypertensive rat (SHR) and the $\mathrm{BN}$ rat [19] have also been reported in this region of rat chromosome 8 . These observations led us to extend the phenotype screening of our congenics beyond the variables characterising Nidd/gk5 in the GKxBN F2 cross.

We initially focused our investigations on the congenic strain BN.GK8b, which was designed to cover both regions of chromosome 8 , linked to insulin secretion and body weight. When compared with $\mathrm{BN}$ controls, rats of the BN.GK8b strain exhibited significantly greater body weight and enhanced acute insulin secretion induced by glucose in vivo, thus validating the characteristics of the $\mathrm{Nidd} / \mathrm{gk} 5$ originally defined in the GKxBN F2 cross. Further investigations showed that plasma concentration of TC, HDL-C, LDL-C, TG and PL are significantly greater in BN.GK8b rats than in BN controls. Almost all effects were consistent in males and females and were reproduced in 12 and 24week-old congenic rats. In order to initiate the genetic dissection of this chromosomal interval, we repeated an identical phenotype screening in rats of the congenic strain BN.GK8a, which share with the strain BN.GK8b GK alleles in a $<8.1 \mathrm{cM}$ region of the QTL. Rats of the strain BN.GK8a exhibited significantly enhanced acute insulin response to glucose in vivo, as previously observed in BN.GK8b rats. In contrast, plasma lipid profile was similar between BN.GK8a rats and $\mathrm{BN}$ controls, with the exception of the concentration of plasma $\mathrm{TG}$, which was significantly higher in BN.GK8a rats.

Overall, our results suggest that GK variant(s) in the 6.9 to $8.1 \mathrm{cM}$ congenic region shared between BN.GK8a and BN.GK8b strains control acute insulin secretion and plasma TG levels (Fig. 2). We named this locus Ins $/ g k 1$. Providing that a single gene or a series of closely linked genes at the QTL Ins/gkl control insulin secretion, cholesterol metabolism is regulated by gene variant(s) contained in the chromosomal region of GK origin specific to the congenic strain BN.GK8b (approximately $20 \mathrm{cM}$ between markers D8Got49 and D8Rat30), which we named Chol/gkl (Fig. 2). However, altered plasma lipid levels and enhanced insulin secretion in BN.GK8b rats are likely to be the result of a combined effect of GK variants at the loci Ins/gkl and Chol/gkl. Investigations in a congenic strain designed for Chol/gkl should allow the determination of the specific roles of the two loci in the regulation of insulin secretion and lipid metabolism.

QTL mapping experiments in hybrid populations identify genomic regions defined by statistical estimates, whereas congenic strains allow the definition of the pathophysiological signature of disease susceptibility genes in a well-defined genomic region $[7,8,9,10$, $20]$. Altered phenotypes observed in our congenics are the result of the effects of gene variants localised in the chromosomal interval targeted in the congenics, which are expressed regardless of major influences of GK diabetes susceptibility loci identified in other chromosomes [5, 6]. Essentially, GK variant(s) at the locus Chol/gkl induce a global rise in plasma lipid concentration and a general stimulation of cholesterol metabolism, which may underlie differences between GK and BN strains and not necessarily pathophysiological mechanisms related to diabetes in the GK rat. This hypothesis is supported by the fact that glucose intolerance was the sole criterion used in the production of the GK strain through selective breedings [3]. The fact that $\mathrm{Chol} / \mathrm{gkl}$ coincides with a locus linked to cholesterol metabolism in a BN-SHR strain combination [19] and that high blood pressure was the sole parameter used to derive the SHR strain, may also indicate an 
effect of gene(s) underlying strain differences. In addition, at the rat obesity locus $O b s 3$, alleles originating from the control F344 strain contribute to increased fat pad weight, which may therefore be unrelated to obesity in the OLETF rat [17].

In contrast, the effects of GK gene variants at the locus Ins/gkl on both hyper insulin response to glucose and hypertriglyceridaemia potentially underlie pathophysiological mechanisms. Lipid abnormalities such as elevated fasting plasma TG levels, which are surrogates for TG-rich lipoproteins, and reduced HDL-C concentrations are commonly observed in Type 2 diabetes, hyperinsulinaemia and insulin resistance [21]. Further investigations in congenic substrains carrying shorter GK haplotypes at Ins/gkl will determine whether these phenotypes are controlled by a single gene or by different genes. These congenics will provide useful tools to study the relationship between hyperinsulinaemia and hypertriglyceridaemia in a well-defined genetic context.

Rat gene annotation is rapidly progressing with the availability of the first draft of the rat genome sequence (http://hgsc.bcm.tmc.edu/projects/rat/; http:// www.ensembl.org/Rattus_norvegicus/). Integrating congenic intervals in rat genome sequence data allows a selective search for potential candidates that may participate in altered biological pathways and mechanisms characterised in congenic strains. Interestingly, the gene encoding hepatic lipase (Lipc) is localised in Ins $/ g k l$, and a cluster of genes involved in the regulation of plasma lipoprotein metabolism, including Apo $A I, A I V, A V$ and $C I I I$, map to Chol/gkl. A major fraction of the genetically determined variation in plasma HDL-C in humans is due to allelic variation of the LIPC and APO AI/CIII/AIV genes [22]. We have analysed sequences from these genes but found no functional polymorphisms between the GK and $\mathrm{BN}$ rats that can account for the phenotypic differences in plasma lipid levels between BN.GK8b congenic rats and BN.GK8a and BN controls. However, we cannot rule out the existence of functionally relevant polymorphisms in non-coding regions of the genes that have not been screened in our study.

Comparative genome analysis data showed that Ins/gkl and Chol/gkl are conserved with a region of mouse chromosome 9, previously characterised for the obesity QTL Dob2 and Mob8 [23, 24, 25], and human chromosomes 11q22.3-24.3, 15q21.2-25.1 and $6 \mathrm{p} 21.1-\mathrm{q} 14.1$. The region of human $15 \mathrm{q}$, which contains the locus LIPC and is associated with variations in HDL-C concentrations in Mexican Americans [26], seems to be homologous to Chol/gkl. It is, however, distinct to the locus of human $15 \mathrm{q}$, influencing plasma TG in the same population [27]. In addition, a locus on human chromosome $15 \mathrm{q}$ near CYP19 is linked to increased susceptibility to Type 2 diabetes in Mexican Americans [28]. Though Cyp19 itself is contained within the Chol/gkl region, the peak of linkage may be up to $40 \mathrm{cM}$ wide and does not exclude the region conserved with $I n s / g k l$ as well. Linkages to variables related to cholesterol metabolism and risk factors for coronary heart disease have also been proposed in regions of 11q23. A locus linked to high TC and ApoB levels [29] appears homologous to Ins/gkl, whereas the region associated with low HDL-C in Utah pedigrees [30] maps $10 \mathrm{cM}$ distal to the APO AI/CIII/AIV cluster and is therefore outside the region of Ins/gkl conserved with human chromosome 11 .

In conclusion, we have refined the localisation of Nidd/gk5 in the GKxBN F2 cross and associated different patterns of pathophysiological characteristics to two regions of the QTL. Congenic strains derived for the locus provide powerful novel models for testing the phenotypic contribution of a well-defined subset of GK alleles in the regulation of insulin secretion and cholesterol metabolism. Further investigations into lipoprotein composition and insulin secretion mechanisms in these and new congenic strains will be crucial for identifying diabetes susceptibility genes at the locus.

Acknowledgements. This work is supported by the Wellcome Cardiovascular Functional Genomics Initiative (066780) and Diabetes UK (RD01/0002160). The production of congenic strains of the GK rat was supported by grants from Diabetes UK (RD96/0001270) and the EC (GIFT, QLRT-1999-00546). S. C. Collins is the recipient of a Wellcome Prize Studentship. D. Gauguier holds a Wellcome Senior Fellowship in Basic Biomedical Science (057733).

The authors thank C. Hetherington (Biomedical Services, University of Oxford, UK) for invaluable help with the implementation of the congenic colonies. R. H. Wallis and K. J. Wallace contributed equally to this work.

\section{References}

1. Hanson RL, Knowler WC (2003) Quantitative trait linkage studies of diabetes-related traits. Curr Diab Rep 3:176-183

2. Horikawa Y, Oda N, Cox NJ et al. (2000) Genetic variation in the gene encoding calpain-10 is associated with type 2 diabetes mellitus. Nat Genet 26:163-175

3. Goto Y, Suzuki KI, Sasaki M, Ono T, Abe S (1988) GK rat as a model of nonobese, noninsulin-dependent diabetes. Selective breeding over 35 generations. In: Shafrir E, Renold AE (eds) Frontiers in diabetes research. Lessons from animal diabetes II. Libbey, London, pp 301-303

4. Portha B, Serradas P, Bailbe D, Suzuki KI, Goto Y, Giroix MH (1991) $\beta$ cell insensitivity to glucose in the GK rat, a spontaneous non obese model for type II (non insulindependent) diabetes. Diabetes 40:486-491

5. Galli J, Li LS, Glaser A et al. (1996) Genetic analysis of non insulin dependent diabetes mellitus in the GK rat. Nat Genet 12:31-37

6. Gauguier D, Froguel P, Parent V et al. (1996) Chromosomal mapping of genetic loci associated with non-insulin dependent diabetes in the GK rat. Nat Genet 12:38-43

7. Gauguier D, Samani NJ (2002) Approaches to the analysis of complex quantitative phenotypes and marker map construction based on the analysis of rat models of hypertension. In: Camp N, Cox A (eds) Quantitative trait loci, methods and protocols. Humana Press, Totowa, pp 225-251 
8. Nadeau JH, Singer JB, Matin A, Lander ES (2000) Analysing complex genetic traits with chromosome substitution strains. Nat Genet 24:221-225

9. Rogner UC, Avner P (2003) Congenic mice: cutting tools for complex immune disorders. Nat Rev Immunol 3:243-52

10. Cowley AW Jr (2003) Genomics and homeostasis. Am J Physiol Regul Integr Comp Physiol 284:R611-R627

11. Bihoreau MT, Sebag-Montefiore L, Godfrey RF et al. (2001) A high resolution consensus linkage map of the rat integrating radiation hybrid and genetic maps. Genomics 75:57-69

12. Collins SC, Wallis RH, Wallace K, Bihoreau MT, Gauguier D (2003) Marker Assisted Congenic Screening (MACS): a database tool for the efficient production and characterisation of congenic lines. Mamm Genome 14:350-356

13. Watanabe TK, Bihoreau M-T, McCarthy LC et al. (1999) A radiation hybrid map of the rat genome containing 5,255 markers. Nat Genet 22:27-36

14. Mu JL, Naggert JK, Svenson KL et al. (1999) Quantitative trait loci analysis for the differences in susceptibility to atherosclerosis and diabetes between inbred mouse strains C57BL/6J and C57BLKS/J. J Lipid Res 40:1328-1335

15. Doerge RW, Churchill GA (1996) Permutation tests for multiple loci affecting a quantitative character. Genetics 142:285-294

16. Lander ES, Green P, Abrahamson J et al. (1987) MAPMAKER: an interactive computer package for constructing primary genetic linkage maps of experimental and natural populations. Genomics 1:174-181

17. Ogino T, Wei S, Wei K et al. (2000) Genetic evidence for obesity loci involved in the regulation of body fat distribution in obese type 2 diabetes rat, OLETF. Genomics 70:19-25

18. Okuno S, Watanabe TK, Ono T et al. (1999) Genetic determinants of plasma triacylglycerol levels in (OLETF x BN) x OLETF backcross rats. Genomics 62:350-355

19. Bottger A, Lith HA van, Kren V et al. (1996) Quantitative trait loci influencing cholesterol and phospholipid phenotypes map to chromosomes that contain genes regulating blood pressure in the spontaneously hypertensive rat. J Clin Invest 98:856-862
20. Rapp JP (2000) Genetic analysis of inherited hypertension in the rat. Physiol Rev 80:135-172

21. Reaven GM (2002) Multiple CHD risk factors in type 2 diabetes: beyond hyperglycaemia. Diabetes Obes Metab 4:S13-S18

22. Cohen JC, Wang Z, Grundy SM, Stoesz MR, Guerra R (1994) Variations at the hepatic lipase and apolipoprotein $\mathrm{AI} / \mathrm{CIII} / \mathrm{AIV}$ loci is a major cause of genetically determined variation in plasma HDL cholesterol levels. J Clin Invest 94:2377-2384

23. West DB, Goudey-Lefevre J, York B, Truett GE (1994) Dietary obesity linked to genetic loci on chromosomes 9 and 15 in a polygenic mouse model. J Clin Invest 94: 1410-1416

24. Mehrabian M, Castellani LW, Wen PZ et al. (2000) Genetic control of HDL levels and composition in an interspecific mouse cross (CAST/Ei x C57BL/6J). J Lipid Res 41: 1935-1946

25. Wang X, Paigen B (2002) Quantitative trait loci and candidate genes regulating HDL cholesterol. A murine chromosome map. Arterioscler Thromb Vasc Biol 22:1390-1401

26. Almasy L, Hixson JE, Rainwater DL et al. (1999) Human pedigree-based quantitative-trait-locus mapping: localization of two genes influencing HDL-cholesterol metabolism. Am J Hum Genet 64:1686-1693

27. Duggirala R, Blangero J, Almasy L et al. (2000) A major susceptibility locus influencing plasma triglyceride concentrations is located on chromosome $15 \mathrm{q}$ in Mexican Americans. Am J Hum Genet 66:1237-1245

28. Cox NJ, Frigge M, Nicolae DL et al. (1999) Loci on chromosomes 2 (NIDDM1) and 15 interact to increase susceptibility to diabetes in Mexican Americans. Nat Genet 21: 213-215

29. Pajukanta P, Terwilliger JD, Perola M et al. (1999) Genomewide scan for familial combined hyperlipidemia genes in finnish families, suggesting multiple susceptibility loci influencing triglyceride, cholesterol, and apolipoprotein B levels. Am J Hum Genet 64:1453-1463

30. Kort EN, Ballinger DG, Ding W et al. (2000) Evidence of linkage of familial hypoalphalipoproteinemia to a novel locus on chromosome 11q23. Am J Hum Genet 66:18451856 